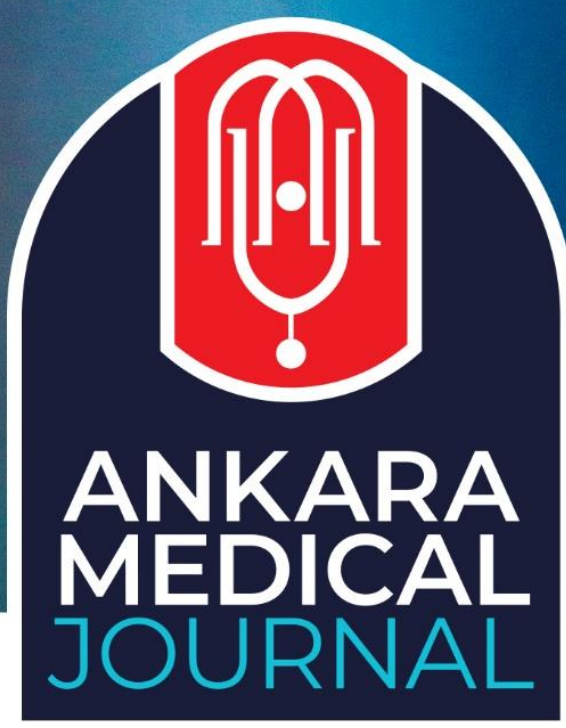

Research Article

Ankara Med J, 2020;(1):90-104 // đot 10.5505/amj.2020.35761

\title{
FACTORS ASSOCIATED WITH DEPRESSION, ANXIETY AND STRESS LEVELS AMONG MEDICAL STUDENTS
}

\section{TIP FAKÜLTESİ ÖĞRENCİLERİNDE DEPRESYON, ANKSIYYETE VE STRES DÜZEYİ İLE İLIŞKILİ FAKTÖRLER}

(1) Naime Meriç Konar ${ }^{1}$

${ }^{1}$ Kirsehir Ahi Evran University, Faculty of Medicine, Department of Biostatistics and Medical Informatics

Yazışma Adresi / Correspondence:

Naime Meriç Konar (e-posta: naime.konar@ahievran.edu.tr)

Geliş Tarihi (Submitted): 23.10.2019 // Kabul Tarihi (Accepted): 12.02.2020

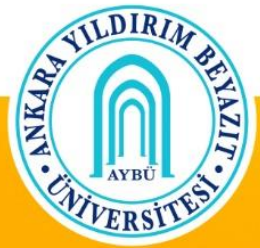

Ankara YIIdrrm Beyazıt University Faculty of Medicine 


\title{
Öz
}

Amaç: Tıp fakültesinde okuyan öğrencilerin yoğun tıp eğitimi ve diğer kişisel faktörler nedeniyle depresif, kaygılı ve stresli olduğu gözlenmektedir. Bu çalışmada Kırşehir Ahi Evran Üniversitesi Tıp Fakültesi ögrencilerinin 1.Kurul Sınavı öncesi ve sonrasında depresyon, anksiyete ve stres prevalansları ve bu düzeylere etki eden faktörlerin belirlenmesi amaçlanmıștır.

Materyal ve Metot: Ekim 2018 - Kasım 2018 tarihleri arasında 1.Kurul Sınavı öncesi ve sonrasında gerçekleștirilen bu çalışmada 42 soruluk Depresyon, Anksiyete ve Stres ölçeği ve akademi, sağlık ve sosyoekonomik bilgileri içeren soruların bulunduğu Genel Bilgi Formu kullanılmıștır.

Bulgular: Totalde, sınav öncesi depresyon, anksiyete ve stres prevalansları sırasıyla $\% 34,04, \% 48,94, \% 32,62$; sinav sorası ise $\% 43,71, \% 49,67$ ve $\% 37,09$ 'dur. İstatistiksel olarak anlamlı olmasa da sinav öncesinde ve sonrasında en yüksek depresyon ( $p=0,228$ ve $p=0,512)$, anksiyete $(p=0,428$ ve $p=0,083)$ ve stres puanları $(p=0,125$ ve $p=0,853)$ Dönem 2 öğrencilerine aittir. Genel olarak, puanlara göre erkek öğrenciler, kız ögrencilere göre daha depresif $(p=0,044$ ve $p=0,018)$, kaygılı $(p=0,392$ ve $p=0,209)$ ve stresli $(p=0,736$ ve $\mathrm{p}=0,977$ ) bulunmuştur. Hem sınav öncesi hem de sınav sonrası depresyon, anksiyete ve stres düzeyleri sağlıkla ilgili risk faktörleri ile ilişkili bulunmuş, sosyoekonomik risk faktörlerinin ise çoğunlukla stres düzeyleri ile ilişkili olduğu saptanmıştır.

Sonuç: Sınav öncesi ve sınav sonrası kaygı prevalansı yüksek bulunmuştur. Sınav sonrası depresyon, anksiyete ve stres prevalanslarının arttığı belirlenmiş, bu nedenle tıp eğitimi alan öğrenciler için akademik ve sosyal destek sistemlerinin olușturulması önerilmiştir.

Anahtar Kelimeler: Anksiyete, depresyon, tıp eğitimi, prevalans, etken, stres.

\begin{abstract}
Objectives: Medical students are observed to feel depressive, anxious and stressful due to intensive medical education program along with other individual factors. This study aims at investigating the prevalence of depression, anxiety and stress levels and their potential determinant among medical students on before and after the exam in newly-established Medical Faculty of Kirsehir Ahi Evran University in Turkey.

Materials and Methods: This study was carried out on before and after the first Committee Exam by conducting 42-item Depression, Anxiety and Stress Scale with General Information Form that includes questions regarding health, academia and socioeconomic-related factors between October and November 2018.

Results: In total, prevalence of depression, anxiety and stress were found as $34.04 \%, 48.94 \%, 32.62 \%$ and $43.71 \%, 49.67 \%$ and $37.09 \%$ on pre and post-exam, respectively. Even though statistically insignificant, PhaseII students had the highest depression ( $\mathrm{p}=0.228$ and $\mathrm{p}=0.512)$, anxiety ( $\mathrm{p}=0.428$ and $\mathrm{p}=0.083)$ and stress scores $(\mathrm{p}=0.125$ and $\mathrm{p}=0.853$ ) on both pre and post exam. Male students were more likely to feel depressed $(\mathrm{p}=0.044$ and $p=0.018)$, anxious ( $p=0.392$ and $p=0.209)$ and stressed $(p=0.736$ and $p=0.977)$ compared to females in overall in terms of their scores. Depression, anxiety and stress levels were mostly associated with healthrelated factors either pre or post-exam. Socioeconomic determinant was found to be mostly associated with stress levels.

Conclusion: High prevalence of anxiety was observed on both before and after the exam. Depression, anxiety and stress prevalence were found to be increasing on post-exam, therefore academic and social support systems are suggested to be provided for medical students.

Keywords: Anxiety, depression, medical education, prevalence, determinant, stress.
\end{abstract}




\section{Introduction}

Medical education is known to be quite abrasive and demanding because of excessive information in limited time, the necessity of excessive research, not having so much spare time to relax, the frequency of examinations which require knowledge of basic sciences and clinical skills and the necessity of having the completeknowledge of these skills in order to intervene the patient's life in case of emergency. Such stressful and intensive 6-years-long medical education programme and these aforementioned factors along with individual problems (family - friends and financial issues) could cause psychological disorders among medical students such as stress, anxiety and even depression.

Depression is a mental illness which has various symptoms including feeling of guilt, pessimism, low motivation, lack of attention, low self-worth, suicidality, sleep disturbance and inappetence. Anxiety is characterized by worried thoughts, tension, increased blood pressure, respiratory rate, pulse rate, sweating, difficulty of swallowing, dizziness, and chest pain, while stress can be defined as any uncomfortable emotional experience accompanied by predictable biochemical, physiological and behavioral changes. ${ }^{1-2}$ All these psychological disorders can be observed in people regardless of their ages, races, genders therefore they are quite prevalent worldwide. For medical students however, their prevalences are known to be higher than the overall prevalences. The global prevalence of depression among medical students has been found as $28 \%$; on the other hand, high prevalence of anxiety and stress among medical students have been reported over the years in many studies. ${ }^{3-11}$

The aim of this study is to determine the prevalence and associated factors of depression, anxiety and stress status before and after the first Committee-Exam among medical students in Kirsehir Ahi Evran University Faculty of Medicine. It is the first study that analyzes the psychological distress levels among medical students in newly-established medical faculty in Central Anatolia, Turkey.

\section{Materials and Methods}

This study was carried out between October - November 2018 with Phase I, Phase II and Phase III medical students of Kirsehir Ahi Evran University Faculty of Medicine. This faculty was established in 2011 and took its first students in 2015, therefore only Phase I, Phase II and Phase III medical students are being educated in this faculty. Inclusion criteria was defined as being 18 years old or older and being educated in medical faculty of the university; while students whose age was below 18 and who are being educated in other faculties of the university were excluded from the study. 


\section{Ethical considerations}

This study was approved by Kirsehir Ahi Evran University non-interventional Clinical Studies Ethical Committee. (Approval Date : 09/10/2018 - Approval Number : 2018-18/154).

\section{Data collection}

Data was collected via validated Turkish version of 42 -item Depression, Anxiety and Stress Scale (DASS-42) and General Information Form which was created by the researcher by reviewing the existing literature. ${ }^{12-13}$ The form includes academia-related (Scholarship, faculty of medicine preference, satisfied with the education of faculty of medicine, entrance of the faculty of medicine, interested in undergraduate transfer), health-related (Smoking status, sleep duration, chronic disease, psychiatric diagnosis of parents or relatives, regular drug use, psychological support, phobia, satisfied with the physical appearance) socio-demographic-related (Sex, nationality, phase, housing, economic status of the family, satisfied with the city, family's attitude, relationship status, hobby, having problems with friends) questions. Questionnaires and General Form were applied approximately 1-week before and 1-week-after the first Committee-Exam. Students were informed about this study via printed Consent Form, which was given with DAS-42 Scale and General Information Form. This study was conducted with the volunteer participants who gave the consent. The original DASS-42 scores can be categorized from normal to very severe for depression, anxiety and stress. For depression, scores less than 9 are defined as 'normal', 10-13 are 'mild', 14-20 are 'moderate', 21-27 are 'severe' and scores greater than 28 are described 'extremely severe'. For anxiety, scores less than 7 are considered as 'normal', 8-9 are 'mild', 1014 are 'moderate', 15-19 are 'severe' and scores above 20 are 'extremely severe'. Stress scores below 14 are in 'normal' category, 15-18 are 'mild', 19-25 are in 'moderate' category, 26-33 are 'severe' stress and scores greater than 34 are defined as 'extremely severe'. However, two categories were created to determine these levels by merging normal-mild categories and moderate-severe and very severe categories in this study. Therefore the cut-off points are defined as less than 13 are 'normal' and scores greater than 14 are 'depressed'; scores less than 9 are normal; while scores greater than 10 are 'anxious' and scores less than 18 and greater than 19 are described as normal and stressed, respectively.

\section{Statistical analysis}

Mean \pm standard deviation and median, minimum, maximum values were given for quantitative variables, while frequencies and percentages were reported for categorical ones. Kolmogorov-Smirnov Test was used to determine the normality of the quantitative data. t-test, Mann-Whitney U Test and One-Way ANOVA were applied depending of the normality of the quantitative data for group comparisons. Multiple logistic regression was used to identify factors affecting the depression, anxiety and stress levels of the students. Univariate analysis such as t-test, Mann-Whitney U Test and Chi-Square Test were performed before multiple logistic 
regression analysis to identify candidate variables for final model. Variables with a p-value $<0.20$ in the univariate analysis were included to the multiple logistic regression model. Backward Wald was used as a variable selection method. Adjusted Odds Ratios (AOR) along with their 95\% confidence intervals (CIs) were reported for these regression models. SPSS (Statistical Programme for Social Sciences) version 22.0 (IBM Corp. Released 2013. IBM SPSS Statistics for Windows, Version 22.0. Armonk, NY: IBM Corp.) was used for all analysis. Significance level was taken as 0.05 .

\section{Results}

The response rate was 63.51\% (141 out of 222) and 68.01\% (151 out of 222) before and after the exam, respectively. Mean age was $19.41 \pm 1.23$ on pre-exam while it was $19.50 \pm 1.17$ on post-exam. Baseline characteristics of the participants on pre and post exam were given in Table 1.

Either before or after the exam, mean of depression, anxiety and stress scores were higher in Phase II students compared to Phase I and Phase III students (Table 2). Moreover, male students were observed to have higher depression and anxiety scores both before and after the exam compared to females (Table 3).

Results revealed that students mostly feel anxious before and after the exam (48.94\% and 49.67\%, respectively). Depression was the second most common mood among students (34.04\% and 43.67\%, respectively) while stress is the least common one (32.62\% and $37.09 \%$, respectively) on pre- and post-exam. Besides, it's worth to mention that prevalence of depression and stress increased after the exam, the most remarkable increase was observed in prevalence of depression, with increment of about 10\% (34.04\% to $43.67 \%$ ); prevalence of stress also increased (32.62\% to $37.09 \%$ ) while prevalence of anxiety remains the same before and after the exam. Psychological distress prevalences were calculated in terms of gender groups. Results revealed that although statistically insignificant, female students were seem to be more anxious and stressed compared to males on both pre $\left(\chi^{2}=1.774 ; \mathrm{p}=0.412\right)$ and post-exam $\left(\chi^{2}=1.146 ; \mathrm{p}=0.564\right)$; while male students were found to be more depressed than females on either pre- or post-exam. Depression, anxiety and stress prevalences were calculated as $25.77 \%, 43.30 \%$ and $30.93 \%$, respectively for females; while they were found as $34.85 \%, 40.91 \%$ and $24.24 \%$, respectively for males on pre-exam. On post-exam however, psychological distress prevalences for females were calculated as $30.70 \%, 38.60 \%$ and $30.70 \%$ for depression, anxiety and stress, respectively; they were $37.35 \%$ for depression, $37.35 \%$ for anxiety and $25.30 \%$ for stress for male students. On both before and after the exam, depression, anxiety and stress prevalences were found similar in terms of gender groups $\left(\chi^{2}=1.774 ; p=0.412\right.$ for pre-exam and $\chi^{2}=1.146 ; p=0.564$ on after the exam) Furthermore, psychological distress prevalences were also reported in terms of Phase for both pre- and postexam. Results suggested that depression, anxiety and stress prevalences were significantly different across 
severity of psychological distress groups on pre-exam only for Phase-I students $(\mathrm{p}=0.007)$, results were summarized in Table 4 (Table 4).

Cronbach Alpha internal consistency coefficients were calculated for this study in order to determine the consistency of responses. They were found as $0.94,0.87,0.89$ on pre-exam; while they were $0.95,0.91$ and 0.91 on post-exam for depression, anxiety and stress sub-dimensions, respectively. Overall Cronbach Alpha coefficient was 0.96 and 0.97 on pre and post-exam, respectively.

Binary logistic regression results revealed that psychiatric diagnosis of first degree family member was the common determinant for all depression $(\mathrm{AOR}=8.327,95 \% \mathrm{CI}=2.376-29.184 ; \mathrm{p}=0.001)$, anxiety $(\mathrm{AOR}=7.783$, $95 \% \mathrm{CI}=2.227-27.199 ; \mathrm{p}=0.001)$, and stress (AOR = 3.063, 95\% CI = $0.998-9.398 ; \mathrm{p}=0.05)$ status of medical students on pre-exam; while it was satisfied with physical appearance determinant on post-exam for each psychological distress levels. Beside psychiatric diagnosis; sleep duration, smoking status, family's economic status and phobia were found to be significantly associated with depression; they were psychological support, satisfied with physical appearance for stress and sleep duration for anxiety on pre-exam. For the post-exam setting, smoking status and faculty of medicine preference for depression; regular drug use, faculty of medicine preference for anxiety; housing and psychological support for stress were significant factors besides satisfied with physical appearance (Table 5). 
Table 1. Baseline Characteristics

\begin{tabular}{|c|c|c|c|c|c|}
\hline \multirow{2}{*}{ Variables } & \multicolumn{2}{|c|}{ Pre - exam } & \multicolumn{2}{|c|}{ Post - exam } & \multirow{2}{*}{$\mathbf{p}$} \\
\hline & $\mathrm{n}$ & $\%$ & $\mathrm{n}$ & $\%$ & \\
\hline \multicolumn{6}{|l|}{ Sex } \\
\hline Female & 87 & 61.70 & 96 & 63.57 & 0.417 \\
\hline Male & 54 & 38.30 & 55 & 36.43 & \\
\hline \multicolumn{6}{|l|}{ Nationality } \\
\hline Turkish & 130 & 92.20 & 141 & 93.38 & 0.822 \\
\hline Other & 11 & 7.80 & 10 & 6.62 & \\
\hline \multicolumn{6}{|l|}{ Relationship Status } \\
\hline Single & 138 & 97.87 & 149 & 98.68 & 0.675 \\
\hline Other & 3 & 2.13 & 2 & 1.32 & \\
\hline \multicolumn{6}{|l|}{ Phase } \\
\hline Phase I & 81 & 57.44 & 74 & 49.01 & 0.344 \\
\hline Phase II & 30 & 21.28 & 40 & 26.49 & \\
\hline Phase III & 30 & 21.28 & 37 & 24.50 & \\
\hline \multicolumn{6}{|l|}{ Smoking Status } \\
\hline Yes & 8 & 6.11 & 17 & 11.33 & 0.241 \\
\hline No & 115 & 87.78 & 127 & 84.67 & \\
\hline Former Smoker & 8 & 6.11 & 6 & 4 & \\
\hline \multicolumn{6}{|l|}{ Regular Drug Usage } \\
\hline Yes & 17 & 12.06 & 22 & 14.57 & 0.607 \\
\hline No & 124 & 87.94 & 129 & 85.43 & \\
\hline \multicolumn{6}{|l|}{ Housing } \\
\hline Family Home & 9 & 6.38 & 11 & 7.28 & 0.523 \\
\hline Dormitory & 91 & 64.54 & 97 & 64.24 & \\
\hline Student Home & 39 & 27.66 & 43 & 28.48 & \\
\hline Relatives' Home & 2 & 1.42 & - & - & \\
\hline \multicolumn{6}{|c|}{ Economic Status of Family } \\
\hline Bad & 2 & 1.42 & 3 & 2 & 0.892 \\
\hline Moderate & 78 & 55.32 & 80 & 53.33 & \\
\hline Good & 61 & 43.26 & 67 & 44.37 & \\
\hline \multicolumn{6}{|l|}{ Scholarship } \\
\hline Yes & 23 & 16.31 & 22 & 14.57 & 0.698 \\
\hline No & 118 & 83.69 & 128 & 85.43 & \\
\hline \multicolumn{6}{|c|}{ Psychological Support } \\
\hline No & 116 & 82.27 & 124 & 82.12 & 0.630 \\
\hline Former Support & 24 & 17.02 & 24 & 15.89 & \\
\hline Yes & 1 & 0.71 & 3 & 1.99 & \\
\hline \multicolumn{6}{|l|}{ Phobia } \\
\hline Yes & 76 & 53.90 & 76 & 50.33 & 0.542 \\
\hline No & 65 & 46.10 & 75 & 49.67 & \\
\hline \multicolumn{6}{|c|}{ Faculty of Medicine Preference } \\
\hline My preference & 118 & 83.69 & 117 & 77.48 & 0.409 \\
\hline Family's Preference & 13 & 9.22 & 19 & 12.58 & \\
\hline Other & 10 & 7.09 & 15 & 9.94 & \\
\hline \multicolumn{6}{|c|}{ Psychiatric diagnosis (Parents/Relatives) } \\
\hline Yes & 22 & 15.60 & 29 & 19.21 & 0.444 \\
\hline No & 119 & 84.40 & 122 & 80.79 & \\
\hline
\end{tabular}




\begin{tabular}{|c|c|c|c|c|c|}
\hline Yes & 12 & 8.51 & 14 & 9.27 & \\
\hline No & 129 & 91.49 & 137 & 90.73 & 0.840 \\
\hline \multicolumn{6}{|l|}{ Hobby } \\
\hline Yes & 107 & 75.89 & 100 & 66.67 & 0.093 \\
\hline No & 34 & 24.11 & 50 & 33.33 & \\
\hline \multicolumn{6}{|c|}{ Satisfied with the education of faculty of medicine } \\
\hline Yes & 34 & 24.11 & 25 & 16.56 & 0.275 \\
\hline Partly & 83 & 58.87 & 98 & 64.90 & \\
\hline No & 24 & 17.02 & 28 & 18.54 & \\
\hline \multicolumn{6}{|l|}{ Satisfied with the city } \\
\hline Yes & 44 & 31.21 & 36 & 23.84 & 0.189 \\
\hline No & 97 & 68.79 & 115 & 76.16 & \\
\hline \multicolumn{6}{|c|}{ Entrance of the faculty of medicine } \\
\hline First attempt & 83 & 58.87 & 89 & 58.94 & \\
\hline Second attempt & 47 & 33.33 & 57 & 37.75 & 0.214 \\
\hline More than two attempts & 11 & 7.80 & 5 & 3.31 & \\
\hline \multicolumn{6}{|c|}{ Having Problems with Friends } \\
\hline Yes & 12 & 8.51 & 14 & 9.27 & 0.840 \\
\hline No & 129 & 91.49 & 137 & 90.73 & \\
\hline \multicolumn{6}{|c|}{ Satisfied with the physical appearance } \\
\hline Yes & 83 & 58.87 & 78 & 51.66 & 0.461 \\
\hline Partly & 48 & 34.04 & 61 & 40.40 & \\
\hline No & 10 & 7.09 & 12 & 7.94 & \\
\hline \multicolumn{6}{|l|}{ Family's Attitude } \\
\hline Authoritarian & 5 & 3.55 & 9 & 5.96 & 0.178 \\
\hline Indulgent & 76 & 53.90 & 69 & 45.70 & \\
\hline $\begin{array}{l}\text { Either authoritarian or } \\
\text { indulgent }\end{array}$ & 60 & 42.55 & 70 & 46.36 & \\
\hline Uninterested & - & - & 3 & 1.98 & \\
\hline \multicolumn{6}{|c|}{ Interested in undergraduate transfer } \\
\hline Yes & 86 & 60.99 & 97 & 64.67 & 0.545 \\
\hline No & 55 & 39.01 & 53 & 35.33 & \\
\hline
\end{tabular}


Table 2. Depression, Anxiety and Stress Levels in Terms of Phase

\begin{tabular}{|c|c|c|c|c|}
\hline \multirow{12}{*}{ PRE-EXAM } & Depression Score & Mean \pm SD & Median [Min - Max] & p-value \\
\hline & Phase I & & $9[0-41]$ & \multirow{3}{*}{$0.228^{* *}$} \\
\hline & Phase II & & 11.50 [1 - 39] & \\
\hline & Phase III & & $10[0-37]$ & \\
\hline & \multicolumn{4}{|l|}{ Anxiety Score } \\
\hline & Phase I & $10.36 \pm 6.73$ & & \multirow{3}{*}{$0.428^{*}$} \\
\hline & Phase II & $12.23 \pm 7.82$ & & \\
\hline & Phase III & $10.17 \pm 7.76$ & & \\
\hline & \multicolumn{4}{|l|}{ Stress Score } \\
\hline & Phase I & $14.89 \pm 7.48$ & & \multirow{3}{*}{$0.125^{*}$} \\
\hline & Phase II & $18.23 \pm 9.57$ & & \\
\hline & Phase III & $16.87 \pm 7.82$ & & \\
\hline \multirow{12}{*}{ POST-EXAM } & Depression Score & Mean \pm SD & Median [Min - Max] & p-value \\
\hline & Phase I & $12.26 \pm 9.73$ & & \multirow{3}{*}{$0.512^{*}$} \\
\hline & Phase II & $14.15 \pm 87$ & & \\
\hline & Phase III & $11.89 \pm 9.89$ & & \\
\hline & \multicolumn{4}{|l|}{\begin{tabular}{|l|} 
Anxiety Score \\
\end{tabular}} \\
\hline & Phase I & $11.32 \pm 7.91$ & & \multirow{3}{*}{$0.083^{*}$} \\
\hline & Phase II & $13.28 \pm 7.77$ & & \\
\hline & Phase III & $9.27 \pm 7.59$ & & \\
\hline & \multicolumn{4}{|l|}{ Stress Score } \\
\hline & Phase I & $16.15 \pm 8.31$ & & \multirow{3}{*}{$0.853^{*}$} \\
\hline & Phase II & $16.83 \pm 8.72$ & & \\
\hline & Phase III & $15.73 \pm 9.33$ & & \\
\hline
\end{tabular}

*: One Way ANOVA

**: Kruskal-Wallis Test 
Table 3. Anxiety and Stress Levels in Terms of Gender

\begin{tabular}{|c|c|c|c|c|}
\hline \multirow{9}{*}{ PRE-EXAM } & \begin{tabular}{|l} 
Depression Score \\
\end{tabular} & Mean \pm SD & Median [Min - Max] & p-value \\
\hline & Female & & $9[0-41]$ & \multirow{2}{*}{$0.044^{* *}$} \\
\hline & Male & & $11.50[0-37]$ & \\
\hline & \multicolumn{4}{|l|}{ Anxiety Score } \\
\hline & Female & & $9[0-35]$ & \multirow{2}{*}{$0.392^{* *}$} \\
\hline & Male & & $9.50[0-28]$ & \\
\hline & \multicolumn{4}{|l|}{ Stress Score } \\
\hline & Female & $15.84 \pm 8.59$ & & \multirow{2}{*}{$0.736^{*}$} \\
\hline & Male & $16.31 \pm 7.31$ & & \\
\hline \multirow{9}{*}{ POST-EXAM } & Depression Score & Mean \pm SD & Median [Min - Max] & p-value \\
\hline & Female & & $9.5[0-42]$ & \multirow{2}{*}{$0.018^{* *}$} \\
\hline & Male & & $14[1-40]$ & \\
\hline & \multicolumn{4}{|l|}{ Anxiety Score } \\
\hline & Female & & $9[0-42]$ & \multirow{2}{*}{$0.209^{* *}$} \\
\hline & Male & & $11[0-33]$ & \\
\hline & \multicolumn{4}{|l|}{ Stress Score } \\
\hline & Female & $16.24 \pm 9.47$ & & \multirow{2}{*}{$0.977^{*}$} \\
\hline & Male & $16.20+6.99$ & & \\
\hline
\end{tabular}

*: Independent Samples t -Test

**: Mann-Whitney U Test

Table 4. Prevalence of Depression, Anxiety and Stress in Terms of Phase

\begin{tabular}{|c|c|c|c|c|c|c|c|}
\hline Timing & Phase & Severity & Depression & Anxiety & Stress & $x^{2}$ & $\mathbf{p}$ \\
\hline \multirow{6}{*}{ Pre - exam } & \multirow{2}{*}{ Phase I } & Normal or mild & $59(72.84)$ & $41(50.62)$ & $56(69.14)$ & 9.991 & 0.007 \\
\hline & & Moderate, severe and very severe & $22(27.16)$ & $40(49.38)$ & $25(30.86)$ & & \\
\hline & \multirow{2}{*}{ Phase II } & Normal or mild & $17(56.67)$ & $14(46.67)$ & $18(60)$ & 1.496 & 0.473 \\
\hline & & Moderate, severe and very severe & $13(43.33)$ & $16(53.33)$ & $12(40)$ & & \\
\hline & \multirow{2}{*}{ Phase III } & Normal or mild & $17(56.67)$ & $17(56.67)$ & $21(70)$ & 1.165 & 0.559 \\
\hline & & Moderate, severe and very severe & $13(43.33)$ & $13(43.33)$ & $9(30)$ & & \\
\hline \multirow{6}{*}{ Post - exam } & \multirow{2}{*}{ Phase I } & Normal or mild & $43(58.11)$ & $37(50)$ & $48(64.86)$ & 3.358 & 0.187 \\
\hline & & Moderate, severe and very severe & $31(41.89)$ & $37(50)$ & $26(35.14)$ & & \\
\hline & \multirow{2}{*}{ Phase II } & Normal or mild & $20(50)$ & $17(42.50)$ & $25(62.50)$ & 3.270 & 0.195 \\
\hline & & Moderate, severe and very severe & $20(50)$ & $23(57.50)$ & $15(37.50)$ & & \\
\hline & \multirow{2}{*}{ Phase III } & Normal or mild & $22(59.46)$ & $22(59.46)$ & $22(59.46)$ & 0.001 & 0.999 \\
\hline & & Moderate, severe and very severe & $15(40.54)$ & $15(40.54)$ & $15(40.54)$ & & \\
\hline
\end{tabular}


Table 5. Binary Logistic Regression Results

\begin{tabular}{|c|c|c|c|c|c|}
\hline & Dependent Variable & $\begin{array}{c}\text { Adjusted } \\
\text { Odds Ratio }\end{array}$ & $\begin{array}{l}95 \% \text { C } \\
\text { Intery }\end{array}$ & $\begin{array}{l}\text { dence } \\
\text { r AOR }\end{array}$ & \\
\hline & Depression & (AOR) & Lower & Upper & p-value \\
\hline & Sleep duration & 2.316 & 1.506 & 3.560 & $<0.001$ \\
\hline & Smoking Status & & & & \\
\hline & No & Ref. & & & 0.003 \\
\hline & Yes & 4.840 & 1.421 & 16.489 & 0.012 \\
\hline & Former Smoker & 12.321 & 1.896 & 80.054 & 0.009 \\
\hline & Family's Economic Statu & & & & \\
\hline & Bad & Ref. & & & 0.042 \\
\hline & Moderate & 0.053 & 0.002 & 1.801 & 0.103 \\
\hline & Good & 0.024 & 0.001 & 0.848 & 0.040 \\
\hline & Phobia & 2.516 & 1.058 & 5.979 & 0.037 \\
\hline & $\begin{array}{l}\text { Psychiatric diagnosis } \\
\text { (Parents/Relatives) }\end{array}$ & 8.327 & 2.376 & 29.184 & 0.001 \\
\hline & Dependent Variable & & & & \\
\hline & Anxiety & & & & \\
\hline & Satisfied with the physic & earance & & & \\
\hline & Yes & Ref. & - & - & 0.061 \\
\hline & Partly & 1.298 & 0.592 & 2.847 & 0.515 \\
\hline PRE - EXAM & No & 12.856 & 1.511 & 109.390 & 0.019 \\
\hline & Sleep duration & 1.675 & 1.175 & 2.388 & 0.004 \\
\hline & $\begin{array}{l}\text { Psychiatric diagnosis } \\
\text { (Parents/Relatives) }\end{array}$ & 7.783 & 2.227 & 27.199 & 0.001 \\
\hline & Dependent Variable & & & & \\
\hline & Stress & & & & \\
\hline & Psychological Support* & 4.041 & 1.434 & 11.382 & 0.008 \\
\hline & Faculty of Medicine Pref & & & & \\
\hline & My preference & Ref. & - & - & 0.053 \\
\hline & Family's Preference & 3.347 & 0.914 & 12.249 & 0.068 \\
\hline & Other & 3.767 & 0.907 & 15.65 & 0.068 \\
\hline & Phobia & 2.278 & 0.959 & 5.411 & 0.062 \\
\hline & Satisfied with the physic & earance & & & \\
\hline & Yes & Ref. & - & - & 0.033 \\
\hline & Partly & 1.394 & 0.579 & 3.354 & 0.459 \\
\hline & No & 7.800 & 1.667 & 36.49 & 0.009 \\
\hline & $\begin{array}{l}\text { Psychiatric diagnosis } \\
\text { (Parents/Relatives) }\end{array}$ & 3.063 & 0.998 & 9.398 & 0.050 \\
\hline & Dependent Variable & & & & \\
\hline & Depression & & & & \\
\hline & Smoking Status & & & & \\
\hline & No & Ref. & - & - & 0.023 \\
\hline & Yes & 4.863 & 1.081 & 21.869 & 0.039 \\
\hline & Former Smoker & 7.560 & 0.982 & 58.206 & 0.052 \\
\hline & Faculty of Medicine Pref & & & & \\
\hline & My preference & Ref. & - & - & 0.013 \\
\hline & Family's Preference & 5.888 & 1.538 & 22.533 & 0.010 \\
\hline & Other & 3.897 & 0.853 & 17.797 & 0.079 \\
\hline & Chronic Disease & 4.105 & 0.842 & 20.014 & 0.081 \\
\hline
\end{tabular}




\begin{tabular}{|c|c|c|c|c|c|}
\hline \multirow{33}{*}{ POST - EXAM } & Hobby & 0.467 & 0.192 & 1.135 & 0.093 \\
\hline & $\begin{array}{l}\text { Having Problems with } \\
\text { Friends }\end{array}$ & 5.082 & 0.936 & 27.59 & 0.060 \\
\hline & \multicolumn{5}{|c|}{ Satisfied with the physical appearance } \\
\hline & Yes & Ref. & - & - & $<0.001$ \\
\hline & Partly & 4.790 & 1.991 & 11.522 & $<0.001$ \\
\hline & No & 11.484 & 1.809 & 72.912 & 0.010 \\
\hline & \multicolumn{5}{|l|}{ Dependent Variable } \\
\hline & \multicolumn{5}{|l|}{ Anxiety } \\
\hline & Regular Drug Usage & 0.161 & 0.040 & 0.640 & 0.010 \\
\hline & \multicolumn{5}{|c|}{ Faculty of Medicine Preference } \\
\hline & My preference & Ref. & - & - & 0.013 \\
\hline & Family's Preference & 2.733 & 0.837 & 8.924 & 0.096 \\
\hline & Other & 7.140 & 1.639 & 31.113 & 0.009 \\
\hline & $\begin{array}{l}\text { Psychiatric diagnosis } \\
\text { (Parents/Relatives) }\end{array}$ & 2.599 & 0.911 & 7.414 & 0.074 \\
\hline & \multicolumn{5}{|c|}{ Satisfied with the physical appearance } \\
\hline & Yes & Ref. & - & - & $<0.001$ \\
\hline & Partly & 5.886 & 2.608 & 13.283 & 0.114 \\
\hline & No & 3.507 & 0.739 & 16.65 & 0.641 \\
\hline & \multicolumn{5}{|l|}{ Dependent Variable } \\
\hline & \multicolumn{5}{|l|}{ Stress } \\
\hline & \multicolumn{5}{|c|}{ Satisfied with the physical appearance } \\
\hline & Yes & Ref. & - & - & 0.012 \\
\hline & Partly & 2.388 & 1.073 & 5.317 & 0.033 \\
\hline & No & 7.242 & 1.573 & 33.335 & 0.011 \\
\hline & \multicolumn{5}{|l|}{ Housing } \\
\hline & Family Home & Ref. & - & - & 0.014 \\
\hline & Dormitory & 1.639 & 0.353 & 7.607 & 0.528 \\
\hline & Student Home & 0.371 & 0.067 & 2.069 & 0.258 \\
\hline & Psychological Support & 4.022 & 1.442 & 11.218 & 0.008 \\
\hline & \multicolumn{5}{|l|}{ Smoking Status } \\
\hline & No & Ref. & - & - & 0.088 \\
\hline & Yes & 3.692 & 1.112 & 12.257 & 0.033 \\
\hline & Former Smoker & 0.661 & 0.073 & 5.982 & 0.712 \\
\hline
\end{tabular}

\footnotetext{
*: Psychological Support variable was taken as two-level (yes-no)
}

\section{Discussion}

High prevalence of anxiety was found among medical students on pre (48.94\%) and post-exam (49.67\%) in overall. Similar results have been reported earlier.7-10, 14-17 However prevalence of depression $34.04 \%$ $43.71 \%)$ and stress $(32.62 \%-37.09 \%)$ were remarkably lower compared to anxiety prevalence on both pre and post-exam. 
In our study, mean of anxiety scores were found to be higher than mean of both depression and stress scores in all Phases (Phase-I, Phase-II and Phase-III) on pre-exam. Phase-II students had higher psychological distress scores than Phase I and Phase III students. Similar ${ }^{18,19}$ and different results ${ }^{7,9}$ were reported in previous publications in this regard. Moreover, it was found that male students had higher depression and anxiety scores than female students in pre and post-exam. Several researches had been published based on the similar findings as this study. ${ }^{8-10,15,19}$ For the stress scores however, pre-exam scores of male students were higher than females whereas on post-exam scores of female students were higher. Similarly, regardless of the time of the examination, mean of depression and anxiety scores were found to be higher and stress scores were lower in males than females in a previous study. ${ }^{9}$ On the other hand, in contrast to our results, higher psychological distress levels among female students has been in many studies. 4, 5, 10, 18, 20-23, 24

Moreover, unlike our results, housing was found as a determinant for anxiety ${ }^{10}$; on the other hand, living alone was not observed as a determinant for depression. ${ }^{4}$ Similarly, smoking status was reported as a determinant for depression ${ }^{9}$, financial support was found to be a determinant for stress, depression and anxiety ${ }^{8}$ and this factor was thought to be similar as economic status of the family factor in our study. Moreover, living with family was observed to be positively associated to depression and anxiety levels, while it was negatively associated with stress levels ${ }^{8}$ Furthermore, in contrast to our results, having chronic disease was found to be associated with depression. ${ }^{21}$

Results of this study pointed out that especially Phase-II students are facing high levels of anxiety regardless of the time of the exam, this could be the result of intensive medical education syllabus of this Phase compared to syllabuses of Phase I and Phase III along with the frequency of exams which are being applied throughout the academic year. Another reason for this issue would be that Phase III education is known to be the introduction of clinical years after the completion of two-years-long syllabus containing basic medical sciences. Therefore, Phase III students are believed to be more involved to the medical education after either the two years of the education experience or the syllabus's more clinic-based contents. Phase I syllabus is not so intensive as the syllabus of Phase II and Phase III, hence students of this Phase are observed to be more relax and also they are thought to be in resting compared to Phase II and Phase III students because of the feeling of confidence of the entrance to the Faculty of Medicine.

In the current study, depression and stress prevalence did not seem to be in alarming level for the students especially on pre-exam. One reason for this result would be that this study was conducted before and after the first Committee-Exam of Phase-I, Phase-II and Phase-III; therefore contents of these exams were not so intensive. However, results demonstrated that all of the students, regardless of their Phase were observed to have high levels of anxiety both before and after the exam. This result is perturbative with regard to being already a high prevalence of anxiety since its levels are expected to increase throughout the academic year. 
Hence, our suggestion would be to make arrangements in order to minimize the anxiety levels of students such as readjust the syllables so as to distribute especially the contents of the Phase - I and Phase - II equally, establishing academic and social support systems which enables the students to help lessen their anxiety levels.

The questionnaire and the DAS-42 Scale were applied before and after 1-week of the first Committee-Exam, therefore depression, anxiety and stress levels, their prevalence and factors associated to these psychological distress levels could be affected by other factors rather than the exam itself. For this reason it's suggested that inventories regarding these psychological distress levels should be applied to the students at the time of and right after the exam, to be able to have the clear insight of their prevalence, the real psychological distress levels and real factors affecting to them. Furthermore, depression, anxiety and stress levels were measured on only one pre and post-exam. Therefore, the other suggestion would be to apply these questionnaire and DAS-42 Scale on every pre and post- Committee-Exam to be able to evaluate the change in these psychological distress levels of medical students within the academic year more effectively.

This study has some limitations. Firstly, it was conducted on a newly-established medical faculty which has only Phase I, Phase II and Phase III students. Therefore, psychological distress levels of medical students on clinical years (Phase-IV, Phase-V, Phase-VI) of the faculty could not be analyzed. Secondly, it's a single-center study therefore these results cannot be generalized to the whole medical-student population of Turkey. Thirdly, part of this study is questionnaire-based therefore bias cannot be ignored. Moreover, it should be noted that approximately $40 \%$ of the students did not answer the questionnaire both on pre and post exam in total, this might be due to the timing of the application of this questionnaire, that is, questionnaires were asked to fill in during the courses, thus high absence rate of the courses was seemed to affect the response rate of the study. Furthermore, the design of this study is cross-sectional therefore casual relationships could not be determined. For this reason, the associations found in this study cannot be evaluated as in the form of casual nature. Further longitudinal studies should be performed in order to examine that kind of relationship.

To conclude up, a comprehensive study was conducted for evaluating the prevalence and associated factors of depression, anxiety and stress levels among medical students in Turkey. Further research that could cover the whole medical student population of Turkey is needed in order to determine the overall prevalence and related factors for these psychological distress levels. 


\section{References}

1. American Psychiatric Association. Diagnostic and statistical manual of mental disorders, 5th ed. American Psychiatric Pub;2013.

2. Baum A. Stress, Intrusive Imagery, and Chronic Distress, Health Psychol 1990;6:653-75.

3. Puthran R, Zhang MW, Tam WW, Ho RC. Prevalence of depression amongst medical students: a metaanalysis. Medical Educ 2016; 50(4): 456-68.

4. Mayer FB, Santos IS, Silveira PS et. al. Factors associated to depression and anxiety in medical students: a multicenter study. BMC Med Educ 2016;16(1):282.

5. Al Saadi T, Addeen SZ, Turk T, Abbas F, Alkhatib, M. Psychological distress among medical students in conflicts: a cross-sectional study from Syria. BMC Med Educ 2017;17(1):173.

6. Gazzaz ZJ, Baig M, Al Alhendi BSM et.al Perceived stress, reasons for and sources of stress among medical students at Rabigh Medical College, King Abdulaziz University, Jeddah, Saudi Arabia. BMC Med Educ 2018; 18(1):29.

7. Iqbal S, Gupta S, Venkatarao E. Stress, anxiety \& depression among medical undergraduate students \& their socio-demographic correlates. Indian J Med Res 2015;141(3):354.

8. Fuad MDF, Lye MS, Ibrahim N, Ismail SIFB, Kar PC. Prevalence and determinants of stress, anxiety and depression among preclinical medical students in Universiti Putra Malaysia in 2014. Int J Collab Res Intern Med Public Health 2015;7(1):1.

9. Kulsoom B, Afsar NA. Stress, anxiety, and depression among medical students in a multiethnic setting. Neuropsychiatr. Dis. Treat 2015;11:1713-22.

10. Abdallah AR, Gabr HM. Depression, anxiety and stress among first year medical students in an Egyptian public university. Int Res J Med Sci 2014;2(1):11-9.

11. Ramya N, Reddy R, Ranganath TS, Subathra VA cross sectional study to assess the psychological morbidity (depression, anxiety, stress) among undergraduate medical students in Bangalore Medical College and Research Institute, Bengaluru. Int J Community Med Public Health 2018;5(9):4103-6.

12. Bilgel N, Bayram N. Turkish version of the Depression Anxiety Stress Scale (DASS-42): psychometric properties. Noro Psikiyatr Ars 2010;47(2):118-27.

13. Lovibond PF, Lovibond SH. The structure of negative emotional states: Comparison of the Depression Anxiety Stress Scales (DASS) with the Beck Depression and Anxiety Inventories. Behav Res Ther 1995;33(3):335-43.

14. Wahed WYA, Hassan SK. Prevalence and associated factors of stress, anxiety and depression among medical Fayoum University students. Alexandria J. Med 2017;53(1):77-84.

15. Patil SP, Sadhanala S, Srivastav MU, Bansode, Gokhe SS. Study of stressors among undergraduate medical students of a teaching medical institution. Int J Community Med Public Health 2017;4:3151-4. 
16. Fawzy M, Hamed SA. Prevalence of psychological stress, depression and anxiety among medical students in Egypt. Psychiatry Res 2017;255:186-94.

17. Yusoff MSB, Rahim AFA, Baba AA, Ismail SB, Pa MNM. Prevalence and associated factors of stress, anxiety and depression among prospective medical students. Asian J Psychiatr 2013;6(2):128-33.

18. Sun L, Sun LN, Sun YH et. al. Correlations between psychological symptoms and social relationships among medical undergraduates in Anhui Province of China. Int J Psychiatry Med 2011;42(1):29-47.

19. Coker AO, Coker OO, Sanni, D. Sociodemographic correlates and symptoms of depression, anxiety and stress among a sample of nigerian medical students. Niger J Basic Clin Sci 2018;15(1):58.

20. Chaudhry MA, Khan IA, Ashraf MZ, Ibrahim MT, Mahmood A, Zeb A. Prevalence of Anxiety and Depression among Medical Students of Private Medical College in Pakistan. Biomedica 2017;33(2):103-6.

21. Ngasa SN, Sama CB, Dzekem BS et.al. Prevalence and factors associated with depression among medical students in Cameroon: a cross-sectional study. BMC Psychiatry 2017;17(1):216.

22. Dyrbye LN, Thomas MR, Shanafelt TD. Systematic review of depression, anxiety, and other indicators of psychological distress among US and Canadian medical students. Acad Med 2006;81(4):354-73.

23. Shaikh BT, Kahloon A, Kazmi M et al. Students, stress and coping strategies: a case of Pakistani medical school. Educ Health (Abingdon) 2004;17(3): 346-53.

24. Adhikari A, Dutta A, Sapkota S, Chapagain A, Aryal A, Pradhan A. Prevalence of poor mental health among medical students in Nepal: a cross-sectional study. BMC Med Educ 2017;17(1):232. 